\title{
Effects of repeated exposure to a humanoid robot on children with autism
}

\author{
B. Robins, K. Dautenhahn, R. te Boekhorst, and A. Billard
}

\section{Introduction}

This work is part of the Aurora project which investigates the possible use of robots in therapy and education of children with autism (Aurora 2003), based on findings that people with autism enjoy interacting with computers, e.g. (Powell, 1996). In most of our trials we have been using mobile robots, e.g. (Dautenhahn and Werry, 2002). More recently we tested the use of a humanoid robotic doll. In (Dautenhahn and Billard, 2002) we reported on a first set of trials with 14 autistic subjects interacting with this doll. In this paper we discuss lessons learnt from our previous study, and introduce a new approach, heavily inspired by therapeutic issues. A longitudinal study with four children with autism is presented. The children were repeatedly exposed to the humanoid robot over a period of several months. Our aim was to encourage imitation and social interaction skills. Different behavioural criteria (including Eye Gaze, Touch, and Imitation) were evaluated based on the video data of the interactions. The paper exemplifies the results that clearly demonstrate the crucial need for long-term studies in order to reveal the full potential of robots in therapy and education of children with autism.

\subsection{Autism}

Autism here refers to the term Autistic Spectrum Disorders with a range of manifestations of a disorder that can occur to different degrees and in a variety of forms (Jordan, 1999). The exact cause or causes of autism is/are still unknown. Autism is a lifelong developmental disability that affects the way a person communicates and relates to people around them. People with autism often have accompanied learning disabilities. According to the National Autistic Society (NAS 2003) people with autism have impaired social interaction, social communication and imagination. This can show itself in difficulties in social relationships, the inability to relate to others in meaningful ways, difficulty with verbal and non-verbal communication and in the development of play and imagination. Usually people with autism show little reciprocal use of eye-contact and do rarely get engaged in interactive games. Autism affects more males than females (NAS 2003).

\subsection{Imitation and the Case of Autism}

Imitation plays an important part in social learning both in children and adults. From birth, imitation plays a critical role in the development of social cognition and communication skills, helping an infant in forging links with other people (Nadel et al., 1999). Imitation and turn taking games are used in therapy to promote better body awareness and sense of self, creativity, leadership and the taking of initiative both in children and adults (as used in Dance Therapy by Kalish 1968, Levy 1988, Payne 1990). There are currently contradictory findings in respect of imitative deficits in autism. Some researchers suggest autism-specific impairments in imitation (Rogers and Pennington, 1991; Meltzof and Gopnik, 1993) whilst others show that autistic children are able to engage in immediate imitation of familiar actions (Hammes and Langdel, 1981).

Nadel explored the use of imitation as a communicative means in infant with autism (Nadel et al., 1999) and found significant correlation between imitation and positive social behaviour. Her findings indicate that imitation is a good predictor of social capacities in children with autism. In addition, it was also found that autistic children improve their social responsiveness when they are being imitated (Dawson and Adams 1984, Tiegerman and Primavera, 1981, Nadel et al.,1999). In therapy too, imitation, reflection and synchronous movement work has been used with autistic children to develop social interactions (Costonis 1974, Adler 1968). 


\subsection{The Aurora Project}

In most of the trials conducted within the Aurora project we have been using mobile robots, e.g. (Dautenhahn and Werry, 2002). More recently we tested the use of a humanoid robotic doll, called Robota (Billard, 2003). In (Dautenhahn and Billard, 2002) we reported on a first set of trials with 14 autistic subjects. The central theme of these trials was imitation games between the robot and the children. A camera system analysed gross arm movements of the children that in turn could trigger the robot to imitate the child. Also, Robota performed movements on its own in order to encourage the children to mirror the robot's movements. It was thus hoped to initiate imitative interaction games between Robota and the children. However, the results were inconclusive, and we identified a number of drawbacks of the original setup. Firstly, the set up required the children to sit still at a table, facing the robot, and moving their arms in a very distinct manner, due to limitations of state of the art vision systems that cannot identify subtle movements. Secondly, the children's participation in the interaction games substantially depended on explicit encouragement by a teacher who sat next to them. Overall, our experiences showed that the particular set up did not seem to facilitate the emergence of spontaneous, proactive, and playful interaction games. Lastly, in these previous trials each child was only exposed once to the robot, a situation were accidental parameters can potentially have a significant effect on the interactions observed. A small number of exposures to the robot is also not likely to give any indications with regards to any therapeutic or educational effects. For the purpose of the present study we therefore decided a) to use a much more unconstrained set up, posing only very little constraint on the children's behaviours and postures that are allowed during the interactions, b) to pursue a longitudinal study and expose each child a number of times to the robot, and c) to reduce the intervention of carers so as to focus on spontaneous and self-initiated behaviour of the children.

\section{The Research Questions}

The primary aim of this paper is to investigate to what extent repeated exposure to a humanoid robot, over a long period of time, can help to increase basic social interaction skills in children with autism. Also, varieties of interactions that can be observed will be documented.

\subsection{Longitudinal Research}

As mentioned above, the longitudinal repeated measure design reduces the influence of variables that could lead to 'accidental outcomes', because the same subjects are used. For example, we noticed that unplanned changes in the schedule of activities prior to a trial, such as cancelling the school's assembly, can significantly affect the children's behaviour because of the change to their routine. Also in longitudinal studies there are fewer cases of random variation to obscure the effects of the experimental conditions.

It is very common in therapy to design programmes of intervention/treatment to take place over a period of a year or longer, where, for example, 50 or more sessions of Art Therapy are not unusual (Evans and Dubowski 2001), or in Dance Movement therapy (Seigel 1984, Adler 1968) where case studies show that it might take six months or more for the first breakthrough in the interaction between the therapist and an autistic child to occur.

Similarly, in education there is increasing use of the Qualification and Curriculum Authority's (QCA's) P-scales assessment method (QCA 2003) to assess pupils' performance and to support monitoring of progression and target setting for pupils with learning difficulties. This is usually done once a year and although in many cases the pupils move up a level at the end of a year, often pupils show very slow progress in some developmental areas and stay at the same level for more than a year, simply covering more ground at that level.

A common approach in therapy involves the therapist to gradually attune to the client. This slow process reduces anxiety and distress levels and allows the gradual development of the therapeutic relationship. For these reasons, and because of the long term projection that is used in education, we designed our trials to take place over a longer period of time. On the one hand we wanted to minimize the anxiety and distress the autistic children might find themselves in, caused by a change of routine, being in a novel situation with a new and unusual toy (the robot), and a new person (the investigator). On the other hand we wanted to allow enough time for the children to use any interaction skills they might have (e.g. 
Effects of repeated exposure to a humanoid robot on children with autism

3

eye-contact, turn-taking, imitation), in a reassuring environment, where the predictability and repetitive behaviour of the robot is a comforting factor. Furthermore, we intended to allow enough time and opportunity for the children to improve their social interaction skills by attempting imitation and turn-taking games with the robot while slowly increasing the unpredictability of the robot's actions. We also wanted to be able to monitor the children's reaction to different appearances of the robot, cf. (Ferrara and Hill 1980) study where children with autism play with different non-robotic toys. In our study this involved two different appearances of the robot, namely a 'pretty girl doll' as opposed to plain clothing with a featureless head (the comparison of these two experimental conditions is beyond the scope of this paper and will be discussed in a separate publication).

Overall, this approach has been designed to allow the children to have unconstrained interaction with the robot with a high degree of freedom, on their terms to begin with (providing it is safe for the child and safe for the robot), and to build a foundation for further possible interactions with peers and adults using the robot as a mediator (Werry et al., 2001).

\section{The Trials}

The trials took place in Bentfield Primary school in Essex, UK, a mainstream school with approximately 220 typically developing pupils. The school also has an Enhanced Provision unit to cater for nine pupils with various learning difficulties and physical disabilities. These pupils, each accompanied by a carer, pursue their own unique curriculum and are integrated in the mainstream classes, according to their age group. They participate in any class activity that they are able to.

\subsection{The Set Up}

The trials were conducted in the Light \& Sound room at the school. This is a familiar room for the children, as they often use it for various activities. The Light and Sound area, which is an extended part of the room, was closed off by a curtain leaving a large empty area of approximately $5.5 \mathrm{~m} \mathrm{x} 4.5 \mathrm{~m}$, with a carpeted floor. The room had one door and several windows overlooking the school playgrounds.

The robot was connected to a laptop and placed on a table against the wall at one side of the room. Two stationary video cameras were placed in the room, one at the side to capture the area in front of the robot and the children when approaching the robot, and the other camera placed behind the robot to try and capture the facial expressions of the children as they interacted with the robot in close proximity. We felt that having manned cameras (with yet more adult strangers in the room) would be too intrusive and would cause additional stress to the children. However, despite having two cameras in most of the trials, there were periods of time when the children moved outside the range of the cameras, as the nature of the trials gave them the freedom to move around in the large room. 


\subsection{The Robot}

The robot used in these trials is Robota - a $45 \mathrm{~cm}$ high, humanoid robotic doll (Billard, 2003). The main body of the doll contains the electronic boards (PIC16F870, $4 \mathrm{MHz}$ and $16 \mathrm{~F} 84,16 \mathrm{MHz}$ ) and the motors that drive the arms, legs and head giving 1 DOF to each. The robot has also the capability of being connected to various sensors such as infrared emitters/ receivers, light detectors and more, which were not used in these trials. The arms, legs and head of the robot are plastic components of a commercially available doll. The robot can react to touch by detecting passive motion of its limbs and head through its potentiometers. For a complete description of Robota's hardware see (Billard, 2003).

Robota is connected through a serial link to a PC and can use speech synthesis, speech processing and video processing of data from a quick-cam camera. Using its motion tracking system, Robota can copy upward movements of the user's arms, and sideways movements of the user's head when the user sits very still and close to the robot, looking straight at it, engaging in turn-taking and imitation games with the robot. Machine learning algorithms allow Robota to be taught e.g. a sequence of actions as well as a vocabulary.
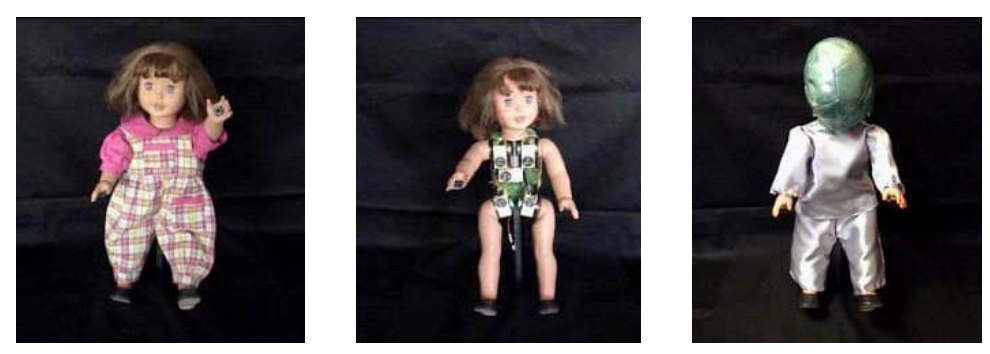

Figure 1. The robot in its two different appearances (the centre figure shows the 'undressed' version revealing the robotic parts that control its movement)

Robota has orginally been developed as a robotic toy that supports a rich spectrum of multi-modal interations with typically developing children, involving speech, music and movements. However, all of the behavioural qualities required in situations of social interaction are less natural to children with autism. Such qualities would include: being still, having a long enough focus of attention, and maintaining gaze on another's face. These are advanced tasks for these children to perform as it lies directly in one of the main areas of their impairment - communication and social interaction. Therefore, in the current trials, Robota's features of speech processing, motion tracking, and learning were not used. As explained above the trials are designed to be unconstrained, with minimal structure, to allow the children to have the greatest degree of freedom. Possibly other features of Robota could be used in future experiments where we will slowly introduce more structure and complexity into the trials, allowing the children time to build their confidence and increase their social interaction skills according to their abilities.

In the current set of trials, the robot has been programmed to operate in two basic modes:

a) as a 'dancing toy' where it moved its arms, legs and head to the beat of pre-recorded music. We used three types of music - children's rhymes, pop music and classical music, following the teacher's advice as to the children's liking.

b) as a puppet, whereby the investigator is the puppeteer and moves the robot's arms, legs or head by a simple press of buttons on his laptop.

\subsection{The Children}

Four autistic children age 5-10 from the Enhanced Provision unit at Bentfield primary school were selected by their teacher to participate in the trials. Each child participated in as many trials as was possible for him during that period (nine trials each on average). The children are:

E.M. - Age 5, in the Reception class. E.M. uses only two or three words but is beginning to communicate using the Picture Exchange Communication System (PECS).

B.B. - Age 6, in year one. B.B. has some limited verbal expression which he uses to express some needs, likes and dislikes. He understands simple directions associated with routines. 
Effects of repeated exposure to a humanoid robot on children with autism

5

B.S. - Age 10 , in year 5. B.S. has autism combined with severe learning difficulties. He has no verbal language and uses symbols and signs to make choices and to express basic needs. He will generally have a go at whatever task he is presented with unless he is feeling unwell when his behaviour deteriorates.

T.M. - Age 10 , in year 5 . He has verbal language which he may use to express needs but often elects not to do so. He can be very difficult to motivate and it is sometimes very difficult to channel his attention towards a particular task

Once a year the school assesses the pupils' performance using the QCA's P-scale method. It is important to view the children's behaviour during the trials in the context of their personal development level which was assessed by their teacher six months prior to the trials.

According to the assessment of their personal and social development level, in the subject of attention, E.M and B.B have been assessed at a level where they pay rigid attention to their own choice of activity, and are highly distractible in activities or tasks led by others. B.S and T.M have been assessed at a level where they can attend to an adult directed activity but require one to one support to maintain their attention. In the area of interacting and working with others, E.M. was assessed at a level where he engages in solitary play or work and shows little interest in the activities of those around him. B.B, B.S. and T.M. were assessed at level where they might take part in work/play with one other person and take turns in simple activities with adult support.

\subsection{Trial Procedures}

Before each trial, the robot was placed on a table ready to start with a click of a button from the laptop. The investigator was sitting next to this table operating the laptop when necessary. The cameras, operated by a remote control, were set to 'standby" mode ready to record.

The children were brought to the room by their carer, one at a time. Each trial lasted as long as the child was comfortable with staying in the room. The trials stopped when the child indicated that he wanted to leave the room or if he became bored after spending 3 minutes already in the room. The average duration of trials was approximately three minutes. A few of the trials lasted up to five minutes, a few others were just under three minutes, and two ended very shortly after they started when the children left the room after 40 and 60 seconds.

The trials were designed to progressively move from very simple exposure to the robot to more complex opportunities for interaction. There were three phases to this:

Setup A - During the first three trials, the robot was placed inside a large open box painted black inside, similar to a puppet-show setting (see figure 2). At this stage in the trials the robot was operating in its 'dancing' mode moving its limbs and head to the rhythm of pre-recorded music. This was simply intended to attract the children's attention to the robot. The children mostly watched while sitting on the floor or on a chair but occasionally left the chair to interact with the robot more closely, (watching closely, touching etc).

This section of the trials was designed mainly for the children to familiarise themselves with the robot (a new toy) and so the carer gave no instructions or tasks for the children to do, simply minimal verbal encouragement if and when this was needed (e.g. 'look, there, what is it?' etc). The children were left to do what they chose to do. The carer and the investigator were generally only observing, intervening only if the child was about to harm the robot (i.e. pushing/pulling the robot's limb using excessive force). The investigator did not initiate communication or interaction with the child, but did respond when addressed by the child.

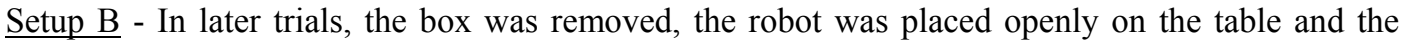
children were actively encouraged to interact with the robot. In this stage the carer introduced physical encouragement, standing with the child near the robot and moving the child's limbs to show him how the robot could imitate his movement (see figure 2). The children could then continue the interaction with the robot on their own. In this situation the robot was operating in its 'puppet mode', where the investigator as puppeteer caused the robot to accurately respond to the child's arm, leg and head movements (even 
when the child was not facing the robot directly or was not in close proximity to the robot). Note, that the investigator's control of the robot was hidden from the children.

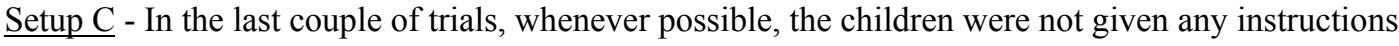
or encouragement to interact with the robot, and were left to interact and play imitation games on their own initiative if they chose to do so. On these occasions the robot was operated as a puppet by the investigator again. The investigator was able to recognise even subtle expressions of the child and to quickly respond to the child's movements, and also to introduce further complexity of turn-taking and role-switch into the simple imitation game.

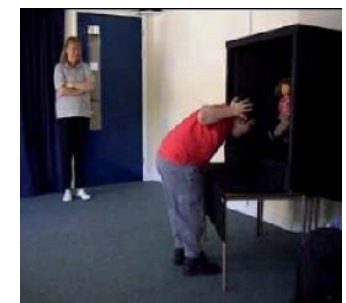

setup $A$

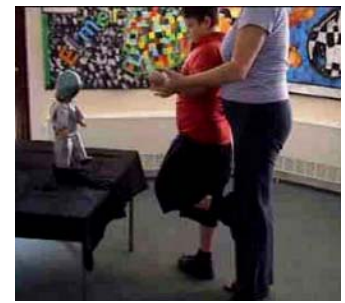

setup $B$

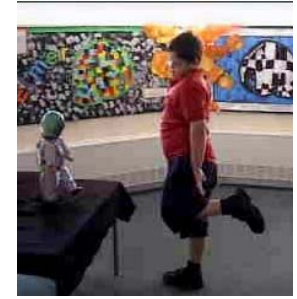

setup $C$

Figure 2. The three phases of the trials

\section{Data Processing and Analysis}

In our trials we defined four elementary behaviour criteria that we evaluated throughout the period of trials, based on the video footage. These behaviours were:
a. Eye Gaze (when directed at the robot)
b. Touch (when the child touched any part of the robot)
c. Imitation (this included direct imitation of the robot's movements, delayed imitation and response to the robot's movement, and attempted imitation of the robot's movement)
d. Near (this included the child approaching the robot and staying in close proximity to the robot regardless of the child's other behaviours)

\subsection{Quantitative Analysis}

The video data from each and every trial for a given child was segmented into one second intervals. The trials were coded by scoring the above defined elementary behaviours every second of the trial, cf. (Tardiff et al., 1999; Dautenhahn and Werry, 2002). The scores for each trial were then summed up and yielded the total number of occurrences of each behaviour during a specific trial and the total duration the child was engaged in each behaviour during that trial. The trials varied in duration, therefore the duration of a behaviour was standardised by expressing it as a proportion of the trial duration.

This data analysis produced various graphs showing changes in the children's behaviour (during the child robot interaction) over a period of time. For each child we followed the trend of each of their behavioural criteria from day one, when the first trial took place, to day 101 when the last trial was conducted.

The graphs in figure 3 give samples of the results. Figure 3 (left) shows that the values for the behaviours of Touch, Imitation and Near all increase considerably towards the later trials, i.e. from day 92 onward. For Eye Gaze highest scores occur during the first two trials on day 1 and day 8 . This could be attributed to the novelty of the situation and due to the fact that the child was given a chair to sit in front of the robot to watch this new toy. Naturally the high score for Eye Gaze can be expected in this situation. However if we disregard these first two trials, we see that the trend for Eye Gaze, too, increases from the third trial onwards, resulting in a relatively high score on the last trial on day 101. 
Effects of repeated exposure to a humanoid robot on children with autism
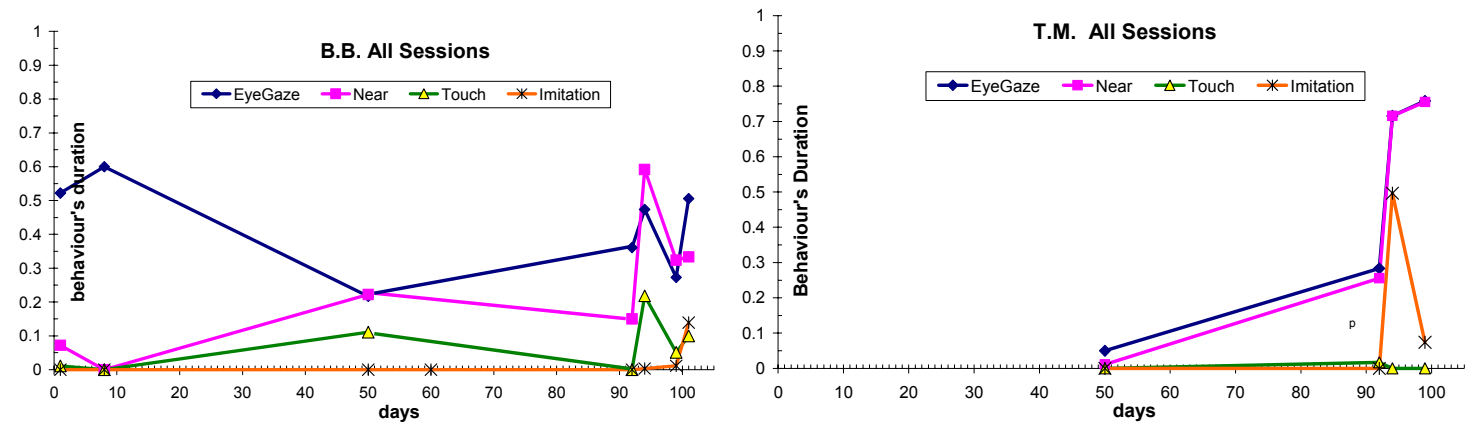

Figure \#. 3. The graph shows the scores for the four behavioural criteria during all the trials that B.B (left) and T.M (right) participated in

Figure 3 (right), which shows the behaviour criteria of T.M. during the trials, demonstrates a considerable increase of the scores for Near, Eye Gaze and Imitation toward days 92 and 94. Touch, although with a very low score, also occurred only on day 92.

The data that we have processed also allowed us to monitor each behaviour criteria separately, over the whole period of the trials, across all the children. The graphs in figure 4 show samples of these results.
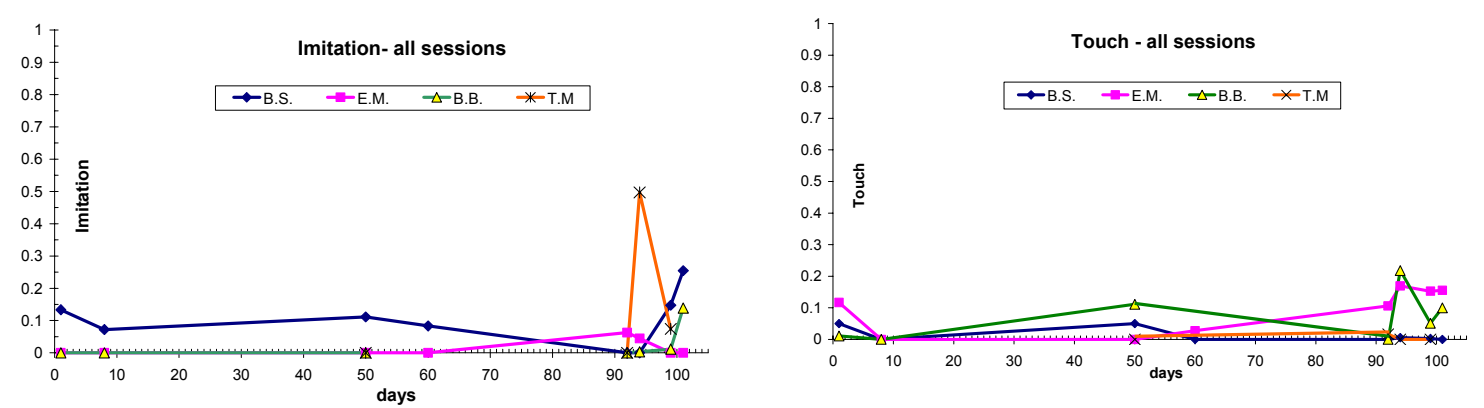

Figure 4. The trends of scores for Imitation (left) and Touch (right) throughout the investigation period

Figure 4 (left) shows a trend of Imitation scores as it appeared in all children throughout all the trials with a visible increase at the end of the trial period - from day 92 onwards. In figure 4 (right) the Touch scores increase for some of the children in the last trials, days $92-101$.

\subsection{Qualitative Analysis}

As stated earlier, one of the overall questions that we are investigating within this project is whether exposure to and interaction with the robot help increase the autistic child's social interaction skills using imitation and turn-taking games for this purpose. During the analysis of the video recordings of this set of trials we noticed several occasions when the children also interacted with the adults in the room (i.e. their carer, or the investigator). Sometimes this occured in relation to the robot, when the robot acted as a 
mediator or an object of shared attention, but at other times these interactions were not robot related. To understand the events that take place in such interactions requires attention to the autistic child's activities in their interactional context. The quantitative analysis alone, based on the frequency and duration of the basic behaviours, cannot reveal some important aspects of social interaction skills (imitation, turn-taking, role-switch) and the communicative competence that the autistic children showed during the trials.

A comprehensive qualitative analysis of some of those segments of the trials where the children showed such interaction skills and communicative competence is beyond the scope of this paper and will be discussed in a separate publication. However, the following gives a description of a very short segment (duration of 32 seconds) taken from one child's trial on the second to last day, which reveals such interaction skills:

Action Response

1. Robot raises left arm - Child mirrors and raises right arm

2. Robot raises left arm - Child mirrors and raises right arm

3. Robot raises left arm - Child mirrors and raises right arm

4. Robot raises right arm - Child mirrors and raises left arm

5. Robot raises right arm - Child mirrors and raises left arm

6. pause (under $1 \mathrm{sec}$ )

7. Child raises right arm - Robot mirrors and raises left arm

8. Robot raises left arm - Child starts to raise left arm, quickly drops it and raises right arm

9. Child raises left arm - Robot mirrors and raises right arm

10. Robot turns head to the right - Child mirrors and turns head to left

11. Robot turns head to the right - Child mirrors and turns head to left

12. Child shakes head up and down - Robot turns head to left.

13. child pauses

14. Robot raises right arm - Child starts to raise right arm, quickly drops it and raises left arm

We can see that during this segment the child showed the following social interaction skills: a) straightforward imitation of various body parts' movements (lines 1-5, 9-11,14), b) the child realised when he made a mistake in imitation and corrected himself (lines 8,14$)$, c) the child initiated interaction as part of the imitation and turn-taking game without any pre-determined cue thus causing a role-switch (lines 7,9), and d) the child tried to initiate interaction using a new movement - shaking the head up and down. The child indicated a comprehension that this movement is beyond the robot's capability and so moved on without insisting on that movement (line 13). As stated earlier, those skills shown here would not be revealed in a purely quantitative analysis.

\section{Conclusion}

This paper presented a novel study of longitudinal research on the exposure of a humanoid robot to children with autism ${ }^{1}$. Relatively little work has been done on using autonomous robots in autism therapy, cf. (Dautenhahn and Werry, 2002) for a comprehensive overview on related work where usually the same children are only exposed once, or a few times to a robot. In constrast, our current approach of repeated trials over a long period of time allowed the children time to explore the interaction space of robot-human, as well as human-human interaction. In some cases the children started to use the robot as a mediator, an object of shared attention, for their interaction with their teachers (cf. Werry et al., 2001). Furthermore, once they have got accustomed to the robot, in their own time and on their own initiative, they all opened themselves up to include the investigator in their world, interacting with him, and actively seeking to share their experience with him as well as with their carer. We believe that this is an important aspect of the work, since this human contact gives significance and (emotional, intersubjective) meaning to the experiences with the robot. Future work will, for example, address the statistical analysis of the video data, verify the interrater reliability of the method used, as well as continue the development of new interaction games with Robota and other robots we are using.

\footnotetext{
${ }^{1}$ We are grateful to the teaching staff, parents, and children at Bentfield Primary school. Our special thanks go to the headteacher Mr. Draper for his continued support.
} 
Effects of repeated exposure to a humanoid robot on children with autism

\section{References}

Adler, J. (1968) The Study of an Autistic Child, proceeding of the $3^{\text {rd }}$ annual conference of the American Dance Therapy Association 1968, Madison, Wis.

Aurora (2003) The Aurora Project, url: http://www.aurora-project.com last accessed 25/09/03.

Billard, A. (2003) Robota: Clever Toy and Educational Tool. Robotics \& Autonomous Systems 42, 259-269

Costonis, M. (ed.) (1978) Therapy In Motion, University of Illinois Press, Urbana

Dawson, G. and Adams, A. (1984) Imitation and social responsiveness in autistic children, Journal of Abnormal Child Psychology 12, 209-26.

Dautenhahn, K., Billard, A. (2002) Games Children with Autism Can Play With Robota, a Humanoid Robotic Doll. In: S. Keates and P. M. Langdon and P.J. Clarkson and P. Robinson (eds.): Universal Access and Assistive Technology, Springer-Verlag London, pp. 179-190.

Dautenhahn, K., Werry, I., Rae, J., Dickerson, P., Stribling, P., Ogden, B. (2002) Robotic Playmates: Analysing Interactive Competencies of Children with Autism Playing with a Mobile Robot. In: K. Dautenhahn, A. Bond, L. Cañamero, B. Edmonds (eds.): Socially Intelligent Agents - Creating Relationships with Computers and Robots, Kluwer Academic Publishers, pp. 117-124.

Evans, K. and Dubowski, J. (2001) Art therapy with children on the autistic spectrum: Beyond words. Jessica Kingsley Pub., Philadelphia, PA

Ferrara, C., Hill, S. D. (1980) The responsiveness of autistic children to the predictability of social and non-social toys. J. Autism and Developmental Disorders 10(1), pp. 51-57

Hames, J. G and Langdell, T. (1981) Precursors of symbol formation in childhood autism, Journal of Autism and Developmental Disorders 11, 331-44.

Jordan, R. (1999) Autistic Spectrum Disorders - An Introductory Handbook for Practitioners. David Fulton Publishers, London

Kalish. B. (1968) Body Movement Therapy for Autistic Children, proceeding of the $3^{\text {rd }}$ annual conference of the American Dance Therapy Association 1968, Madison, Wis.

Levy, F. (1988) Dance Movement Therapy - A Healing Art, The American Alliance for Health, Physical Education, Recreation and Dance, Reston, VI

Meltzoff, A. and Gopnik, A. (1993) The role of imitation in understanding persons and developing a theory of mind. In S. Baron-Cohen, H. Tager-Flusberg and D. Cohen (eds.), Understanding other minds: Perspectives from autism (p. 335-66). Oxford University Press.

NAS (2003) National Autistic Society UK, url: http://www.nas.org.uk, last accessed 25/09/03.

Nadel, J., Guerini, C., Peze, A. \& Rivet, C. (1999) The evolving nature of imitation as a format of communication, In: J. Nadel and G. Butterworth (Eds.) Imitation in Infancy, Cambridge University Press, pp. 209-234

Payne, H. (1990) Creative Movement and Dance, Winslow Press, Oxon

Powell, S. (1996) The use of computers in teaching people with autism. In: Autism on the agenda: papers from a National Autistic Society Conference. London

QCA (2003) The qualifications and Curriculum Authority, url: http://www.qca.org.uk/ca/foundation/profiles.asp\#p_scales Last accessed 25/11/03

Rogers S. J., Pennington, B. F. (1991) A theoretical approach to the deficits in infantile autism. Development and Psychopathology 3: 137-162

Siegel, E. V. (1984) Dance-movement therapy: mirror of our selves - the psychoanalytic approach, Human Sciences.

Tardiff, C. \& Plumet, M-H. \& Beaudichon, J. \& Waller, D. \& Bouvard, M. \& Leboyer, M. (1995) Micro-Analysis of Social Interactions Between Autistic Children and Normal Adults in Semi-Structured Play Situations. International Journal of Behavioural Development, 18 (4), 727 - 747

Tiegerman, E. and Primavera, L. (1981) Object Manipulation: an interactional strategy with autistic children. Journal of Autism and Developmental Disorders 11, 427-38.

Werry, I., Dautenhahn, K., Ogden, B., Harwin, W. (2001) Can Social Interaction Skills Be Taught by a Social Agent? The Role of a Robotic Mediator in Autism Therapy. In M. Beynon, C. L. Nehaniv, K. Dautenhahn, Cognitive Technology: Instruments of Mind. Proc. CT2001, Springer Verlag, LNAI 2117, pp. 57-74. 


\section{Contact Details}

Title: Effects of repeated exposure to a humanoid robot on children with autism

Authors: Ben Robins, Kerstin Dautenhahn, Rene te-Boekhorst and Aude Billard

Contact details:

Ben Robins

Adaptive Systems Research Group

The University of Hertfordshire,

Department of Computer Science

College Lane, Hatfield, Hertfordshire AL10 9AB, U.K

Tel: 01707281150

Fax: 01707284185

e-mail : b.1.robins@herts.ac.uk 